\title{
Urbane Produktion und temporäre räumliche Nähe in Produktionsprozessen
}

\section{Urban manufacturing and temporary spatial proximity in production processes}

https://doi.org/10.2478/rara-2019-0061

Eingegangen: 09. Januar 2019; Angenommen: 22. Oktober 2019

\begin{abstract}
Kurzfassung: In diesem Beitrag wird argumentiert, dass temporäre räumliche Nähe zwischen Wirtschaftsakteuren neben der Wissensgenerierung ebenfalls einen produktionsbedingten Zweck verfolgen kann. Dabei werden materielle Konsumgüter aufgrund neuer technologischer Entwicklungen und Trends durch temporäre räumliche Nähe zwischen Endkunden und Produzenten erzeugt und in diesem Beitrag am Beispiel der urbanen Produktion diskutiert. Der temporäre Austausch hat das Ziel, ein den individuellen Wünschen der jeweiligen Kunden entsprechendes materielles Gut in Echtzeit zu erstellen. Ziel dieses Beitrags ist es, produktionsbedingte temporäre räumliche Nähe zu analysieren aufzuzeigen, wie sie Produktionsstandorte und Produktionsstätten sowie die Beziehung zu Kunden prägt. Anhand einer Literaturanalyse und Fallstudien wird produktionsbedingte temporäre räumliche Nähe in Großunternehmen, Manufakturen, Makerspaces und sogenannten Festivals untersucht. Die Ergebnisse zeigen, dass Kunden intensiv in den Produktionsprozess eingebunden sind. Veränderungen der Produktionsweise und Produktionsstätte sind die Konsequenz, da es um die Herstellung von Einzelstücken geht. Zusätzlich verändern sich aber auch die Produktionsstandorte, die aufgrund größerer Marktnähe in stark frequentierte Einzelhandelslagen oder Wohngebiete rücken. Produktionsbedingte temporäre räumliche Nähe zwischen Produzenten und Kunden und damit verbundene innerstädtische Produktion wird die massenhafte Herstellung von Konsumgütern nicht ablösen. Sie ist jedoch ein wichtiger Faktor, der die der Wahl von Geschäftsmodellen und Unternehmensstandorten angesichts des gegenwärtigen Trends der Individualisierung von Konsumgütern beeinflusst.
\end{abstract}

Schlüsselwörter: Koproduktion; temporäre räumliche Nähe; urbane Produktion

\begin{abstract}
This article argues that temporary spatial proximity between economic agents can also have a productionrelated purpose besides knowledge generation. At the same time, due to new technological developments and trends, consumer goods are generated by temporary spatial proximity between end customers and producers. The purpose of the temporary exchange is to create material goods meeting the individual demands of the respective customer in real time. The aim of this paper is to analyse production-related temporary spatial proximity of urban manufacturing to show how production sites and locations as well as the relationship with customers are altered. Based on a literature analysis and case studies, production-related temporary spatial proximity in large companies, factories, makerspaces and festivals is examined. The results show that customers are intensively involved in the production process. Changes in the production method and location are the consequence, since it is about the production of individual pieces. In addition, production locations change, as they need to be in retail locations or housing areas, i.e. close to the market where the end consumers are. Production-oriented temporal spatial proximity
\end{abstract}

\footnotetext{
*Corresponding author: Dr. Anna Butzin, Institut Arbeit und Technik, Munscheidstraße 14, 45886 Gelsenkirchen, Deutschland, E-Mail: butzin@iat.eu

Kerstin Meyer, Institut Arbeit und Technik, Munscheidstraße 14, 45886 Gelsenkirchen, Deutschland
} 
between producers and consumers and associated inner-city production will not replace mass production of consumer goods. However, it is an important factor influencing the choice of business models and business locations for entrepreneurs given the current trend in the individualisation of consumer goods.

Keywords: Co-production; Temporary spatial proximity; Urban manufacturing

\section{Einführung}

Eingebettet in die Debatte zur Relevanz verschiedener Proximitätsdimensionen und deren Dynamiken (Boschma 2005; Balland/Boschma/Frenken 2015) wird temporäre räumliche Nähe in der gegenwärtigen Literatur als eine den Wissensaustausch zwischen Wirtschaftsakteuren erleichternde Näheform diskutiert (Bathelt/Henn 2014; Growe 2018a). Messen, Geschäftsreisen, Projekttreffen und Kundenbesuche ergänzen virtuellen Wissensaustausch und Projektarbeit über die Distanz (Bathelt 2017; Growe 2018b). Dadurch ermöglicht temporäre räumliche Nähe Verbindungen zwischen an verschiedenen Orten lokalisierten Produktions- und Innovationssystemen (Bathelt/Henn 2014). Dem Fokus des Wissensaustausches folgend, steht in den vorhandenen Forschungsarbeiten temporäre Nähe in wissensintensiven und serviceorientierten Branchen im Fokus, die durch die Mobilität von Arbeitskräften ermöglicht wird (Lampel/Meyer 2008; Growe 2018b).

Diesem Beitrag liegt die Beobachtung zugrunde, dass temporäre räumliche Nähe zwischen Produzenten und Kunden ebenfalls einen produktionsbedingten Zweck verfolgen kann. Dabei werden materielle Konsumgüter aufgrund neuer technologischer Entwicklungen und Trends durch temporäre räumliche Nähe zwischen Endkunden und Produzenten mithilfe teils mobiler Produktionsmittel (Maschinen, Anlagen, Minifabriken) erzeugt. Der temporäre Austausch hat das Ziel, ein den Wünschen der jeweiligen Kundinnen und Kunden entsprechendes materielles Gut bereits während des Austausches in Echtzeit zu erstellen. Im Gegensatz zum reinen Wissens- und Informationsgewinn sind der Produktionsprozess und die damit verbundene Koproduktion Zweck der temporären räumlichen Nähe. Auf diese Weise entstehen individualisierte Konsumgüter wie Bekleidung, handwerkliche Produkte, Kosmetikartikel, Lebensmittel oder Produkte aus dem 3D-Drucker.

Produktionsbedingte temporäre räumliche Nähe unterscheidet sich von temporärer räumlicher Nähe, die dem reinen Wissensaustausch dient. Mit ihr einhergehende Produktionsprozesse unterschiedlichen Spezialisierungs- und Wertschöpfungsgrades werden als Erlebnis (Pine/Gilmore 1998) ausgestaltet, Kundinnen und
Kunden werden zu Koproduzentinnen und -produzenten und Produktionsstandorte entstehen in Kundennähe. Des Weiteren ist sie Ausdruck zweier aktueller Entwicklungen, der zunehmenden Individualisierung von Massenware und der derzeitigen Maker- und Do-it-yourselfBewegung. Eingebunden in diese beiden mit hohem Veränderungspotenzial einhergehenden Entwicklungen (vgl. Brandt/Butzin/Gärtner et al. 2017), ist produktionsbedingte temporäre räumliche Nähe ein prägender Faktor der Ausgestaltung bzw. Wahl von Geschäftsmodellen, z. B. in Einzelhandel und Handwerk, und Unternehmensstandorten.

Ziel dieses Beitrags ist es, die bisher wenig erforschte produktionsbedingte temporäre räumliche Nähe zu analysieren und anhand von Fallstudien aufzuzeigen, wie sie Produktionsstandorte und Produktionsstätten sowie die Beziehung zu Kundinnen und Kunden prägt. Durch die Analyse werden gleichzeitig räumliche Auswirkungen der Maker- und Do-it-yourself-Bewegung und der Individualisierung von Massenware betrachtet. Die Fallstudien wurden im Rahmen der beiden Forschungsprojekte „Produktion zurück ins Quartier? Neue Arbeitsorte in der gemischten Stadt“ und "ProUrban - Urbane Produktion - zurück in die Stadt?!" erhoben, an denen die Autorinnen beteiligt waren.

Der Beitrag ist wie folgt strukturiert: Im Überblick über den Forschungsstand (Kapitel 2) wird produktionsbedingte temporäre räumliche Nähe anhand der Produktionsstandorte, der Produktionsstätten und der Kundeneinbindung diskutiert. Die darauffolgende Auswahl (Kapitel 3) und Analyse (Kapitel 4) der Fallstudien gliedert sich in Großunternehmen, Manufakturen, Makerspaces und Festivals. Kapitel 5 fasst die Ergebnisse zusammen und im Fazit (Kapitel 6) wird die Produktion in die Diskussion um temporäre räumliche Nähe eingebettet.

\section{Forschungsstand}

In der von Bathelt und Henn (2014) entwickelten Typologie des Wissenstransfers über die Distanz erfährt temporäre räumliche Nähe eine herausgehobene Bedeutung. Temporäre räumliche Nähe ermöglicht die Kombination 
temporärer (z. B. Messen) und permanenter Kontexte (z. B. Betriebe von Kundinnen/Kunden), in denen Wissenstransfer stattfindet. Produktionsbedingte temporäre räumliche Nähe ist von den in der Typologie diskutierten temporären Treffen von Fachgruppen und Geschäftsreisenden den Produzenten-Nutzer-Treffen ähnlich. Der direkte Austausch zwischen technischen Expertinnen/ Experten und Kundinnen/Kunden (face-to-face) zur Aufrechterhaltung der Produktion steht im Vordergrund, wobei die in das Treffen eingebundenen Akteure über komplementäres Wissen verfügen. Das Wissen wird in den verschiedenen Phasen eines Produktionsprozesses, bestehend aus Entwicklung, Materialauswahl, Fertigung und Zusammenfügen von Komponenten, eingebunden. Diese Treffen finden in der Regel in den Produktionsstätten der Unternehmen statt, sodass es sich um temporäre Treffen an fixen Orten anstatt an temporären Orten wie Messen oder Festivals handelt. Die Räumlichkeiten dienen als zusätzliche Informationsquelle (face the place), da Einsicht in verfügbare Ressourcen, Atmosphäre, Arbeitsphilosophie etc. genommen werden kann (Growe 2018b mit Verweis auf Urry 2007; Flögel/ Zademach 2017). Der Ursprung der in der Wirtschaftsgeographie geführten Diskussion um Wissensprozesse, Interaktionen und räumliche Näheformen liegt in der Erforschung eben jenes produktionsrelevanten Wissens, wenn auch mit einem Schwerpunkt auf Produzenten- und Zuliefererbeziehungen (Pavitt 1984; Lundvall 1992). Der derzeitige Schwerpunkt der Diskussion bezieht sich eher auf Wissensprozesse an temporären Orten, verbunden mit der Frage, welchen Einfluss sie auf das Entstehen neuer Märkte haben (Bathelt/Henn 2014; Henn/Bathelt 2015; Schüßler/Grabher/Müller-Seitz 2015). Wissensaustausch im Rahmen produktionsbedingter temporärer räumlicher Nähe findet zwischen Produzentinnen/Produzenten und Kundinnen/Kunden statt. Torre und Rallet (2005: 54) schreiben, dass Face-to-face-Interaktionen den Austausch komplementären Wissens unterschiedlicher Akteure sowie die Lösung von im Innovationsprozess etwaig aufkommenden Konflikten erleichtern. Im Folgenden wird produktionsbedingte temporäre räumliche Nähe anhand folgender Merkmale analysiert: neue Produktionsstandorte, inspirierende und offene Produktionsstätten sowie Kundeneinbindung, Koproduktion und Prosumption.

Diese Merkmale kennzeichnen den Wandel der Geschäftsmodelle der jeweiligen Unternehmen. Nach Bieger und Reinhold (2011: 32) ist ein Geschäftsmodell die "Grundlogik, wie eine Organisation Werte schafft", wobei monetäre und nicht monetäre Werte für die Anspruchsgruppen des Unternehmens (Kunden, Lieferanten,
Personal, Kapitalgeber, Öffentlichkeit etc.) bedeutsam sind. Den sich ändernden Nachfragebedingungen wird dabei in diversen Geschäftsmodellen begegnet, wobei der gemeinsame Nenner unter dem Begriff "Individualisierung" zu fassen ist. So gibt es durch eine verstärkte Ausrichtung auf neue Technologien und Digitalisierung gekennzeichnete Geschäftsmodelle (vgl. Ematinger 2018), die Kundenwünschen durch Einzelstückproduktion begegnen, aber auch solche, die sich auf Handwerkskunst und manuelle Produktionen (rück)besinnen. Auf Produktion ausgerichtete Geschäftsmodelle werden um Dienstleistungsangebote erweitert (Welzbacher/Pirk/ Ostheimer et al. 2015: 21) und damit neue Geschäftsfelder erschlossen (Moritsch 2016: 29). Aber nicht nur produzierende Unternehmen übernehmen Dienstleistungen zur Distribution und den Aufbau eigener Verkaufsstellen und entwickeln sich dadurch zu direkten Konkurrenten ihrer Einzelhandelskunden, welche weiterhin gleichzeitig beliefert werden. Auch Handelsunternehmen strukturieren sich neu, z. B. mit „Kontraktproduktion (Contract Manufacturing) von Handelsmarken bis zur Übernahme der Produktion" (Zentes 2012: 90), wodurch neue Wertschöpfungsstufen erschlossen werden.

\section{Neue Produktionsstandorte}

Standorte produktionsbedingter temporärer räumlicher Nähe werden durch den gegenwärtigen Trend der Do-ityourself- und Maker-Kultur begünstigt, denn „Machen ist in" (vgl. Drotschmann 2010; Wolf/McQuitty 2011; Hirshberg/Dougherty/Kadanoff 2016). Der derzeit deutlichste Ausdruck des Trends sind von neuen Technologien geprägte Orte wie FabLabs oder Makerspaces. Dort können Technikbegeisterte eigene Ideen oder Projekte mithilfe von vor Ort vorhandenen Maschinen austüfteln, in die Anwendung bringen und bedarfsgerecht produzieren (Fox 2013; Meier/Wirth 2013; Hirshberg/Dougherty/Kadanoff 2016; Schmidt/lbert/Kuebart et al. 2016; Cohen/Jones/Smith et al. 2017). Eine ebenfalls starke Do-it-yourself-Ausrichtung haben Kundenworkshops in Baumärkten. ${ }^{1}$ Durch Individualisierung von Massenware rückt Produktion aber auch stärker in die Einzelhandelsstandorte: In IKEA-Häusern, zum Beispiel, stehen von Fachkräften zu bedienende Stickmaschinen bereit, durch die von Kundinnen und Kunden erworbene Textilien wie Handtücher individuell bestickt werden können, ${ }^{2}$ oder es werden Schuhe des Herstellers Keens in Schuh-

1 Vgl. https://www.hornbach.de/cms/de/de/mein_hornbach/mein markt/veranstaltungen/veranstaltungen_listing.html (11.09.2019).

2 Vgl. https://www.ikea.com/de/de/stores/kamen/ (11.09.2019). 
geschäften gefertigt, indem dort eine kleine Fabrik errichtet wird. ${ }^{3}$

Aus räumlicher Perspektive wird dieser Trend unter der Bezeichnung „Urbane Produktion“ analysiert, wobei das Augenmerk auf produzierenden Unternehmen und auf professionellem bzw. organisiertem Do-it-yourself in Makerspaces und auf Maker-Festivals liegt. Urbane Produktion ist die „[...] Herstellung und Verarbeitung von materiellen Gütern in dicht besiedelten Gebieten, die häufig in unmittelbarer Nähe zum Wohnort der Unternehmerinnen und Unternehmer, Mitarbeiterinnen und Mitarbeiter und/oder der Kundinnen und Kunden entstehen“ (Brandt/Butzin/Gärtner et al. 2017: 27). Urban produzierende Unternehmen sind neben den genannten Makerspaces und FabLabs beispielsweise Manufakturen, die sich auf die handwerkliche Herstellung meist hochwertiger Produkte spezialisiert haben, traditionelle Handwerksunternehmen und Lebensmittelbetriebe. Meist handelt es sich um kleine und mittlere Unternehmen. Die oben geschilderten Beispiele (IKEA, Keens) verdeutlichen aber, dass Produktion in Endkundennähe auch von großen Unternehmen betrieben wird. Neue Technologien (3D-Druck, Lasercutter), emissionsarme Produktionsverfahren und die Herstellung von Kleinserien oder Einzelstücken ermöglichen neue, kleinflächige Produktionsstandorte. Durch die Kundennähe sind Unternehmen in der Lage, „Produkte kurzfristig zu designen, zu konfigurieren, herzustellen, aufzubereiten und zu recyceln“ (Scheelhaase/Zinke 2016: 43).

Urbane Produktion erfolgt kleinteilig und stadtverträglich auf dezentralen Produktionsstandorten, deren Attraktivität zukünftig noch weiter steigen könnte. Denn auch die Digitalisierung der Arbeitswelt bzw. der mit Industrie 4.0 einhergehende Produktionswandel bieten Potenziale für innerstädtische Standorte von Branchen, die auf kleiner Fläche kundennah und individualisiert produzieren (Mühl/Busch/Fromhold-Eisebith et al. 2019). Läpple (2013) bezeichnet urbane Produktion als neue, städtische Ökonomie und plädiert in seinem Essay „Produktion zurück in die Stadt?" dafür, Stadtökonomien durch urbane Produktion und lokale Wertschöpfungsketten robuster gegenüber Schwankungen des Weltmarkts zu machen. Innerstädtische Einzelhandelslagen, urbane Wohnquartiere und Mischgebiete sind das Umfeld der Produktionsstätten, wohingegen großflächige Gewerbegebiete aufgrund fehlender Kundennähe eher ungeeignet sind. Damit ist urbane Produktion eine zusätzliche Nutzungskonkurrenz neben Wohnen, Dienstleistungen

$3 \mathrm{Vgl}$. https://gearjunkie.com/keen-robot-builds-uneek-shoes (11.09.2019). und Freizeit für bereits stark nachgefragte, Mischnutzungen erlaubende Standorte. Zudem ist die Stadtverträglichkeit der Produktion eine Herausforderung für die klassische, stadtplanerische Funktionstrennung von Wohnen, Arbeiten und Erholen, da sie Produktion starr und Durchmischung verhindernd wirken lässt (Erbstößer 2016).

\section{Inspirierende, offene Produktionsstätten}

Räumlichkeiten, in denen produktionsbedingte temporäre räumliche Nähe stattfindet, sollen Informationen preisgeben und Offenheit vermitteln. Zusätzlich zu geschultem Personal schaffen sie eine Atmosphäre für Interaktionen, durch die es Kundinnen und Kunden gelingt, ihre neue, aktivere Rolle auszufüllen. Der durch face-the-place hervorgerufene Effekt ist also bereits Teil des Produktionsprozesses. Kundinnen und Kunden sollen Inspiration finden und in kurzer Zeit ihre Vorstellungen über das zu produzierende Gut konkretisieren, zu diesem Zwecke gegebenenfalls mit intuitiv nutzbarer Technik und Maschinen umgehen und den Prozess als ein positives Erlebnis in Erinnerung behalten (face-themoment; vgl. Growe 2018b mit Verweis auf Urry 2007). Damit dies gelingt, sind Räumlichkeiten, in denen Produkte verkauft werden, als Bühne zu verstehen, wodurch der funktionale Kaufzweck in den Hintergrund rückt. Produkte werden emotionalisiert und der Einkauf zum „Erlebniskauf" (Pine/Gilmore 2000). Zur Differenzierung beschreiben andere Autoren den Produktionsprozess als Kundenreise (Lemon/Verhoef 2016; Følstad/Kvale 2018) oder als Kundeninteraktion, während derer Interaktionen in sechs unterschiedliche Phasen gegliedert sind: Kommunikation, Exploration, Konfiguration, Wartezeit und Lieferung, After-Sales und Feedback, Wiederkauf. Drei dieser Phasen können durch temporäre räumliche Nähe in realen Verkaufsumgebungen stattfinden (Reichwald/ Piller 2009: 273). Dazu gehören Exploration (exploring), das heißt, durch Prototypen oder Beispiele sollen Kundinnen und Kunden eine Einschätzung darüber gewinnen, wie das gewünschte Produkt aussehen könnte; Konfiguration, das heißt die aktive Einbindung in den Herstellungsprozess; Wartezeit und Lieferung, das ist die Zeit, in der das Produkt maschinell hergestellt wird, Kunden aber noch im Geschäft sind. Die Kundenreise wird insbesondere in der Literatur zum Customer Experience Management als mehrdimensionale Erfahrung (sensorische, intellektuelle, relationale und emotionale; vgl. Drengner/Jahn 2012) betrachtet, die durch die Ausgestaltung der Räumlichkeiten angeregt werden soll (Verhoef/Lemon/Parasuraman et al. 2009). Damit hat die Produktionsstätte, wenn auch in kleinerem Umfang, 
Ähnlichkeit mit „Brand Lands“ (Mikunda 2006). Dies sind Orte, an denen rund um etablierte Marken oder Produkte Erlebniswelten für Konsumentinnen und Konsumenten, wie z. B. die Autostadt in Wolfsburg oder Maggi-Kochstudios, mit dem Ziel der Kundenbindung geschaffen werden.

Die Offenheit von Produktionsstätten beschreiben Schmidt, Ibert, Kuebert et al. (2016) am Beispiel von Open Creative Labs. Darunter sind Co-working Spaces, Startup Factories, FabLabs, Hacker Spaces oder offene Werkstätten zu verstehen. Open Creative Labs haben demnach mehrere Dimensionen: Zeitlichkeit, soziales Kuratieren, analoge und virtuelle Räumlichkeiten, freie Wissensteilung, Nutzer- und Problemorientierung, Unvollständigkeit und Übergangsorte. Zeitlichkeit bedeutet, dass eine permanente Struktur an einer festen Adresse etabliert ist, die temporär genutzt werden kann. Sozial kuratierte Offenheit lässt prinzipiell jeder und jedem die Möglichkeit, die Räumlichkeiten unter bestimmten Regeln zu nutzen. Die Arbeitsumgebung ist offen gestaltet und soll den Austausch und das Teilen von Wissen fördern. Die Nutzer- und Problemorientierung fokussiert auf das individuelle Erarbeiten und Kontrollieren moderner Fabrikationsabläufe. Unvollständigkeit beschreibt, dass sich diese Räumlichkeiten immer wieder wandeln, sich ihren Kundinnen/Kunden und Besucherinnen/Besuchern anpassen und sich erweitern können. Open Creative Labs sind Übergangsorte, weil Mittel und Zweck neu zusammengebracht werden und einen Karrierewechsel in einen neuen Beruf, z. B. aufgrund neu erlernter Gewerke und Methoden, bedingen können. Einige dieser Punkte finden sich in den nachfolgenden Beispielen wieder und sind Voraussetzung für die im Folgenden beschriebene Kundenintegration.

\section{Kundenintegration, Koproduktion und Prosumption}

Veränderte Kundenbeziehungen und Kundenintegration erfordern einen interaktiven und offenen Produktionsprozess (Huba/McConnell 2006; Nadeau 2006; Reichwald/Piller 2009; Thallmaier 2015). „Die Kernidee einer solchen Kundenintegration in die Wertschöpfung ist, dass durch den Einbezug von Abnehmern bzw. Nutzern in ehemals vom Herstellerunternehmen dominierte Aktivitäten ein Wissenstransfer zwischen den Akteuren stattfindet, der bei einer klassischen Abwicklung der Leistungserstellung nicht möglich ist" (Reichwald/Piller 2009: 42). Letztere zielt auf durchschnittliche Kundenbedürfnisse $a b$ und birgt das Risiko, heterogene Kundenwünsche nicht befriedigen zu können (Reichwald/ Piller 2009: 135). Kundeneinbindung ist folglich von zentraler Bedeutung für Unternehmen, um der zuneh- mend gewünschten Individualisierung von (Massen) ware Rechnung zu tragen. Produktionsprozesse erhalten dadurch eine dienstleistungsorientierte Ausrichtung. Temporäre räumliche Nähe als Instrument, Zugang zum Wissen der Kundinnen und Kunden zu erhalten, ist dabei ein wichtiger Erfolgsfaktor.

Die Generierung von Wissen durch Kundenintegration ist in der wirtschaftsgeographischen Literatur insbesondere Forschungsobjekt in interaktiven, das heißt von verschiedenen Akteuren entwickelten, Innovationsprozessen. Eine für die Kundenintegration in Produktionsprozessen wichtige Unterscheidung ist die Steuerbarkeit des Innovationsprozesses, je nach Art der Kundenintegration (vgl. Grabher/lbert/Flohr 2008: 264 ff.). Geht es um beratende und partizipierende Kundenintegration, im Rahmen derer Kundinnen und Kunden ihr Erfahrungswissen bezogen auf die Nutzung des Produkts oder Prototypen mitteilen, behält das produzierende Unternehmen die Entscheidungshoheit über die Richtung der Innovations(weiter)entwicklung. Hier wird Kundenwissen innerhalb eines eng definierten Bezugsrahmens nachgefragt und trägt inkrementell zur Verbesserung des Produkts aus Nutzersicht bei. Weitaus offener ist die Integration von Wissen aus Anwender- und Interessencommunities, die von Grabher, lbert und Flohr (2008: 262 mit Referenz zu Kunz/Mangold 2004) als „hybrid communities" bezeichnet werden. In „hybrid communities", wie sie etwa in der Softwareentwicklung zu finden sind (z. B. Linux), verschmilzt die Experten- und Laienrolle. Der Verlauf des Innovationsprozesses ist für einen alleinigen Akteur weniger steuerbar und das Spektrum des eingebrachten Wissens weitaus breiter (z. B. technisches, gestalterisches, anwendungsbezogenes Wissen).

Für die Analyse von Produktionsprozessen eignet sich die Einteilung von Kundeneinbindung anhand der Begriffe Ko-Kreation, Koproduktion und Prosumption (Toffler 1981; Humphreys/Grayson 2008; Xie/Bagozzi/ Troye 2008; Wolf/McQuitty 2011; Fox 2013), die durchaus als Analogie zur Kundenintegration in Innovationsprozesse im Sinne von Grabher, lbert und Flohr (2008) interpretiert werden kann. Ko-Kreation erfordert geringere Kundeneinbindung und bezieht sich auf die Nutzung des Produkts nach dem Kauf, denn nur durch Nutzung, z. B. die Anwendung eines Handys, entfaltet sich der Wert des Produkts. Es kann vom Kunden inkrementell weiterentwickelt werden, indem Kundenwissen und -erfahrungen, z. B. in Form von Verbesserungsvorschlägen, Fehlermeldungen und Anregungen, an den Produzenten berichtet werden. Bei Koproduktion hingegen werden vormals vom Unternehmen voll- 
zogene Stufen im Produktionsprozess von Kundinnen und Kunden übernommen. Dazu zählt der Zusammenbau von Möbelteilen nach deren Erwerb, also etwa die Endproduktion im Falle vieler IKEA-Möbel, aber auch die Kundenintegration in den Produktionsprozess, bevor das Produkt erworben wird (Keen). Das Repertoire eingebrachten Kundenwissens ist breiter, da es sich auf unterschiedliche Stufen im Produktionsprozess beziehen kann. Es kann kundenindividuelle Präferenzen beinhalten genauso wie Wissen um den Zusammenbau von Möbeln. Der Produktionsprozess muss daher durch bedienbare Maschinen, handhabbare Materialien oder verständliche Bauanleitungen entsprechend angepasst sein. Prosumption hingegen ist eine Produktionsform, bei der Konsumentinnen und Konsumenten Produkte für den eigenen Konsum herstellen, indem sie (Roh)materialien und Komponenten einer Wertschöpfung zuführen, von der sie profitieren (Xie/Bagozzi/Troye 2008; Wolf/ McQuitty 2011). Das Wissen bezieht sich auf alle Produktionsstufen, wodurch der gesamte Produktionsprozess durch den Bedarf bzw. die Nachfrage der Kundinnen und Kunden gesteuert ist. Prosumption findet vor allem in den Bereichen Energie- und Lebensmittelversorgung statt, aber auch in der Eigenproduktion von Kleidung, Möbeln und Ersatzteilen. Sie wird durch das Eröffnen von Makerspaces begünstigt, da Konsumentinnen und Konsumenten Produkte dort für ihren Eigenbedarf herstellen können. Auch hier können "hybrid communities“ entstehen, in denen aus dem primären Wunsch der Eigenproduktion eine unternehmerische Produktion folgen kann (Brinks/lbert 2015).

Im Folgenden werden neue Produktionsstandorte und Produktionsstätten sowie Kundenintegration, Koproduktion und Prosumption empirisch anhand von Fallstudien analysiert. Die Analyse mündet in eine vergleichende Diskussion der Frage nach den damit verbundenen, neuen Geschäftsmodellen.

\section{Methodik und Auswahl der Fallstudien}

Ausgangslage für die Untersuchung von Fallstudien zu urbaner Produktion war ein vom Ministerium für Heimat, Kommunales, Bau und Gleichstellung Nordrhein-Westfalen in Auftrag gegebenes Forschungsgutachten mit dem Titel „Produktion zurück ins Quartier? Neue Arbeitsorte in der gemischten Stadt", welches vom Institut Arbeit und Technik (IAT) und dem Büro Stadtraumkonzept bearbeitet wurde. Das Forschungsdesign folgte einem eingebet- teten Fallstudiendesign (Yin 2003), durch das Quartier, Standort und Produktionsunternehmen als zu untersuchende Einheiten betrachtet wurden. Die Auswahl der Fallstudien erfolgte nach der obigen Definition urbaner Produktion im Hinblick auf den Standort in unmittelbarer Nähe zu Wohnorten in einem mehrstufigen Prozess. Aus 25 Steckbriefen von in Frage kommenden Fallstudien (Typ der urbanen Produktion, Traditionsunternehmen vs. Neugründung, Räumlichkeit, Fläche, Beschäftigte, Quartierstyp, Art der Nutzungsmischung, Organisationsform, Kundeneinbindung), ermittelt durch eine Medienanalyse, Expertengespräche in Planungsämtern und Organisationen der Wirtschaftsförderung sowie eine Onlinerecherche, wurden in einer als Projektbeirat fungierenden Fokusgruppe, der Expertinnen und Experten aus Ministerien, Kommunen und Wirtschaftsförderung angehörten, Fallstudien zur näheren Untersuchung ausgewählt. Auswahlkriterium dabei war die Diversität der einzelnen Unternehmen sowie der Fokus auf Fallbeispiele aus dem deutschsprachigen Raum mit einem Schwerpunkt auf Nordrhein-Westfalen. Zwei der Fallstudien stammen aufgrund ihrer besonderen Produktionsweise in Minifabriken aus dem US-amerikanischen bzw. asiatischen Raum. Von den so selektierten Fällen sind neun Gegenstand dieser Analyse. Die Unternehmen wurden angefragt und anhand leitfadengestützter Interviews bei einem Besuch mit Führung durch die Produktionsstätte befragt. Zwei Fallstudien konnten aufgrund ihres Standorts in den USA bzw. Asien nicht besucht, sondern ausschließlich durch eine Recherche analysiert werden.

In einem weiteren vom Bundesforschungsministerium geförderten Projekt mit dem Titel „ProUrban Urbane Produktion - zurück in die Stadt?!", das ebenfalls vom Institut Arbeit und Technik durchgeführt wird, ist ein temporärer Ort entstanden, der Austausch von Produzentinnen/Produzenten und Konsumentinnen/ Konsumenten ermöglicht. Darauf aufbauend ist von Die Urbanisten e.V. das „You do - Festival der Urbanen Produktion" im Jahr 2018 ins Leben gerufen worden. Beide Aktionen fließen als Fallstudien in diese Untersuchung mit ein. Sie wurden mittels teilnehmender Beobachtung und Expertengesprächen analysiert.

Die ausgewählten Fallstudien sind in Großunternehmen, Manufakturen, Makerspaces, FabLabs und Festivals unterteilt, die im Folgenden vorgestellt werden. Die Einteilung in diese vier Gruppen erfolgte aufgrund verschiedener Merkmale, die produktionsbedingte temporäre räumliche Nähe in den jeweiligen Gruppen besitzt, und aufgrund der unterschiedlichen, mit den Fällen verbundenen Geschäftsmodelle. Beispielsweise 
ist produktionsbedingte temporäre räumliche Nähe der Großunternehmen durch neue technische Möglichkeiten und Industrie 4.0 geprägt, während die der Manufakturen einen stärkeren handwerklichen bzw. Do-it-yourselfAnteil haben.

\section{Großunternehmen}

Die von großen Unternehmen betriebene Produktion in innerstädtischen Lagen und die damit verbundene temporäre räumliche Nähe zu Kundinnen/Kunden wird anhand der drei Fallbeispiele Adidas Store-Factory, Hewlett Packard Fitstation und der Keen Smallest Shoe Factory vorgestellt. Davon ist die Adidas Store-Factory besucht und durch Interviews und eine Kundenreise (vgl. Kundenintegration und neue Geschäftsmodelle weiter unten) analysiert worden. Es handelte sich dabei um ein drei Monate laufendes Pilotprojekt, das unter anderem vom Bundeswirtschaftsministerium gefördert wurde. ${ }^{4}$ Die Fallbeispiele Fitstation von Hewlett Packard (im Jahr 2018 Pilotprojekt auf dem US-Markt) und die Smallest Shoe Factory von Keen (im Jahr 2018 Werbetour durch die USA und Asien) basieren auf Internetrecherchen. In der Adidas Store-Factory werden zusammen mit Kundinnen und Kunden individualisierte Wollpullover hergestellt und vor Ort innerhalb weniger Stunden maschinell gestrickt. Die Fitstation von Hewlett Packard ist im Rahmen ihrer Pilotierung in Sportgeschäften aufgebaut und produziert maßgenaue Einlegesohlen. Die Smallest Shoe Factory von Keen besteht aus zwei kleineren Robotern, die in Geschäften und teilweise auch in Schaufenstern ein Schuhmodell von Keen innerhalb weniger Minuten herstellen.

\section{Manufakturen}

Manufakturen sind kleine, inhabergeführte Geschäfte, in denen hochwertige Produkte in Kleinserien oder Einzelstücken handwerklich hergestellt werden. Der Konsum der Produkte wird insbesondere durch Nachfrage nach fairen, ökologischen, qualitativ und ästhetisch hochwertigen Produkten, die lokal hergestellt werden, angetrieben (Läpple 2013). Als Fallstudien sind die Marmeladenmanufaktur in Münster, die Textilwerkstatt Liebesgruß und die Schmuckschmiede (beide in Wuppertal) untersucht worden. Die Marmeladenmanufaktur ist als Feinkostmarke eingetragen, da die handgefertigte Marmelade hochpreisig ist und sich deutlich von der üblichen Massenware abgrenzen soll. Liebesgruß beschreibt sich

$4 \mathrm{Vgl}$ https://www.digitale-technologien.de/DT/Redaktion/DE/ Kurzmeldungen/Aktuelles/2017/2017-04-18_ssw_meldung_ zukunft\%20poroduktion.html (12.09.2019). selbst als Textil-Hommage, denn dort werden Stoffe und T-Shirts weiterverarbeitet und aufgewertet (upcycling). Die Schmuckschmiede wurde von einer Goldschmiedin gegründet, die ein ehemaliges Ladenlokal zu einer Werkstatt mit Verkaufsfläche umgewandelt hat.

Offene Werkstätten, FabLabs und Makerspaces

In vielen Städten und Regionen entstehen Makerspaces, FabLabs und offene Werkstätten. Dort werden Menschen zum Selbermachen und Reparieren von Produkten inspiriert. Häufig werden diese Orte durch die öffentliche Hand, Stiftungen oder Privatwirtschaft subventioniert. Im Folgenden wird der Makerspace „Halle 1“ in Gelsenkirchen und die FabLabs "Das Labor e.V." in Bochum sowie die „Dezentrale - Gemeinschaftslabor für Zukunftsfragen“" in Dortmund analysiert. Die Halle 1 in Gelsenkirchen ist an die Westfälische Hochschule angegliedert und in deren Räumlichkeiten untergebracht. Das Projekt besteht seit 2018 und wurde vom Ministerium für Wirtschaft, Innovation, Digitalisierung und Energie des Landes Nordrhein-Westfalen mit 1,6 Mio. Euro subventioniert (o.V. 2018b: 12). Das Labor e.V. in Bochum ist 2014 entstanden und ehrenamtlich organisiert. Überwiegend finden offene Treffen, Workshops, Vorträge und Informationsveranstaltungen statt. Die „Dezentrale - Gemeinschaftslabor für Zukunftsfragen“ in Dortmund existiert seit 2013 und wird durch das Fraunhofer-Institut für Umwelt-, Sicherheits- und Energietechnik UMSICHT mit Sitz in Oberhausen betrieben.

\section{Festivals}

Im Rahmen des Forschungsprojekts „ProUrban“ des Bundesministeriums für Bildung und Forschung (BMBF) entstand im Herbst 2017 eine zweimonatige Zwischennutzung eines Kirchengebäudes im Bochumer Stadtteil Langendreer-Alter Bahnhof. Diese temporäre Bespielung stand unter dem Motto „Festival der Urbanen Produktion - Langendreer selbermachen“. Die ehemalige und zehn Jahre ungenutzte Lutherkirche wurde zum "LutherLAB“. Ein ähnliches Konzept verfolgte das „You do - Festival der Urbanen Produktion“ im Frühjahr 2018 in Dortmund. Unternehmen, Selbstständige und HobbyMaker gaben ihr Wissen auf Workshops und im Rahmen gemeinsamer Produktentwicklungen an Interessierte weiter. Hergestellt wurden unter anderem UpcyclingProdukte wie Bier, Marmelade, Austernpilze, Samenbomben, Lampenschirme aus Restholz, Hocker aus Sperrmüll, Aquaponikanlagen, das heißt Kreislaufanlagen zur Fischproduktion und Pflanzenzucht, oder solche zur Produktion von Schmuck und Palettenmöbeln oder Prototypen aus dem 3D-Drucker. Ziel dabei war, eine 
erhöhte Wertschätzung gegenüber materiellen Produkten zu schaffen, Know-how zu vermitteln und Menschen zum Nachahmen und Selbermachen einzuladen.

\section{Analyse}

\section{Neue Produktionsstandorte}

In den Fallbeispielen der Großunternehmen sollen Endkunden entweder in den Produktionsprozess integriert werden, erforderliche Daten liefern oder zu nutzende Materialien individuell auswählen. Um die dafür erforderliche Kunden- bzw. Marktnähe zu erreichen und Laufkundschaft anzusprechen, werden stark frequentierte (Einzelhandels-)Standorte für die Produktion bevorzugt. Die neue Standortwahl ist Ausdruck der veränderten Geschäftsmodelle, in denen Interaktion mit Kundinnen und Kunden - und damit ein entscheidender Dienstleistungscharakter - maßgeblich für die Ausgestaltung der Produktionsprozesse ist. Diese Erweiterung der Wertschöpfung durch Kundeninteraktion ist an herkömmlichen Produktionsstandorten von Konsumgütern, das heißt an großflächigen, in der Peripherie gelegenen Produktionsanlagen in Osteuropa oder Asien, nicht realisierbar. Daher bezog die Adidas Store-Factory temporär Geschäftsräume im zentral gelegenen Shoppingcenter Bikini-Berlin. ${ }^{5}$ Die Fitstation und die Smallest Shoe Factory sollten im Sporthandel installiert werden (Carballo 2018; o.V. 2018a). Auch die Produktionstechnik wird an die neuen Standorte angepasst, indem sie nicht nur kleiner und sauberer wird, sondern auch Flexibilität (z. B. hinsichtlich Farben und Formen) ermöglicht, durch die individuellen Kundenwünschen begegnet werden kann. Faktisch dürfen seitens der Produktionstechnik keine besonderen Standortansprüche, wie etwa eine erschütterungsfreie Umgebung, große ebenerdige Flächen oder die Genehmigung erhöhter Lärmemissionen, gestellt werden, um bei der Standortwahl in den gefragten Einzelhandelslagen größte Flexibilität zu haben. Folglich sind die Produktionsprozesse durch emissionsarme Technologien und nur wenige Quadratmeter in Anspruch nehmende Minifabriken gekennzeichnet.

Die Manufakturen liegen in gefragten Stadtquartieren, das heißt in Quartieren, die überwiegend durch eine gründerzeitliche Baustruktur, eine junge, urbane Szene und Mischnutzungen (Wohnen, Gastronomie, Dienstleistungen) gekennzeichnet sind. Aufgrund ihrer

5 Vgl. https://www.bikiniberlin.de/de/journal/adidas_knit_for_you_ im_bikini_berlin/ (12.09.2019). hochwertigen Produkte sind die Manufakturen auf eine höhere Kaufkraft ihrer Kundinnen und Kunden angewiesen. Repräsentative Räumlichkeiten mit Laufkundschaft sind wichtig. Die Manufakturen setzen aber gleichzeitig darauf, dass Kundinnen und Kunden für die Qualität der Produkte längere Wege in Kauf nehmen und gezielt dort einkaufen. Auch Onlinehandel ist ein wichtiger Vertriebskanal. Die Manufakturen weisen intensive Verflechtungen zu anderen Unternehmen im Stadtquartier auf, z. B. durch die gegenseitige Nutzung oder Ausstellung der Produkte oder den Aufbau lokaler Wertschöpfungsketten. Im Vergleich zu den Großunternehmen befinden sich die Manufakturen in etwas kostengünstigeren Lagen. Dies hat zum einen damit zu tun, dass sie inhabergeführt sind und keinem finanzstarken Konzern angehören. Zum anderen erfordern die Ansprüche an die Geschäftsräume recht große Flächen, die in 1a-Lagen mit den angebotenen Produkten nicht bezahlbar wären. Die Geschäftsräume müssen sowohl die Ausstellung der Ware ermöglichen, das heißt, es muss mindestens ein repräsentativer vorderer Bereich mit Schaufenster vorhanden sein, als auch die Produktion und die Lagerung der Rohstoffe. Zusätzlich - darauf wird weiter unten eingegangen - bieten alle der analysierten Manufakturen Kundenworkshops an, wodurch ebenfalls weitere Fläche für Arbeitsplätze benötigt wird. Gleichwohl sind die Manufakturstandorte durch hohe Nutzungskonkurrenz gekennzeichnet. Insbesondere die Akteure aus den Segmenten Wohnen und Gastronomie, die ebenfalls als Nutzer der Räumlichkeiten in Frage kommen, sind in der Regel finanzstärker und können Mietpreissteigerungen besser ausgleichen. So laufen die Manufakturen Gefahr, durch die zum Teil von innen mitgestaltete Aufwertung des Stadtquartiers und den derzeit hohen Druck auf dem Wohnungsmarkt von ihren Standorten verdrängt zu werden.

Der Makerspace Labor e.V. ist in einem Mischgebiet in einem Souterrain im Innenhof einer Blockrandbebauung im Zentrum Bochums untergebracht. Zusätzlich finden zwei monatliche Repair Cafés in Ladenlokalen anderer Initiativen statt. Die "Dezentrale“ in Dortmund liegt im Innenhof einer Blockrandbebauung eines Wohngebiets. Die umliegenden Häuser dienen überwiegend dem Wohnen, vereinzelt sind sie Bürostandort. Im Gegensatz dazu befindet sich die Halle 1 direkt auf dem Campus der Westfälischen Hochschule am Stadtrand Gelsenkirchens. Da von den Einrichtungen keine Emissionen ausgehen und der Publikumsverkehr gering ist, gibt es keine Nutzungskonflikte. In mittel- bis langfristiger Sicht sind die Räumlichkeiten der "Dezentrale" allerdings zu klein und eine Erweiterung am aktuellen 
Standort nur begrenzt möglich. Es gibt derzeit die Überlegung, aus einer Erdgeschosswohnung im Vorderhaus ein Ladenlokal zu machen. Die offenen Werkstätten sind in der Kundenakquise nicht auf Laufkundschaft angewiesen, wie die Unternehmen der beiden anderen Fallstudiengruppen. Sie bedienen eine technikaffine Zielgruppe, die durch den Online-Auftritt und andere Werbemaßnahmen von der technischen Ausstattung angesprochen wird und die Werkstätten besucht. Die Offenheit der Werkstätten wird also nicht über die Lage, sondern durch Werbung, die Produktionsstätte und Kommunikation vor Ort vermittelt. Die Standortwahl erfolgt anhand der Präferenzen der jeweiligen Betreiber und der innen zur Verfügung stehenden Räumlichkeiten und ist entsprechend unterschiedlich. Der Makerspace Labor e.V. liegt zentral und ist gut erreichbar, da die Mitglieder des Vereins vom Stadtrand und den umliegenden Stadtteilen nach Bochum einpendeln. Die „Dezentrale“ residiert im Hinterhaus des Hauses, in dem der Betreiber wohnt, und die Halle 1 ist in den Räumlichkeiten der Universität, die sie initiiert hat, untergebracht.

In beiden Festival-Fällen handelt es sich um zwischen- sowie umgenutzte Räumlichkeiten in zentraler Lage der jeweiligen Quartiere. Im LutherLAB wurde eine Kirche im Bochumer Stadtteil Langendreer-Alter Bahnhof genutzt. Bei der Dortmunder "Werkhalle" handelt es sich um ein ehemaliges Werksgebäude der Hoesch Stahl AG im Unionviertel Dortmund. Beide Orte sind in ihre Quartiere eingebettet und gut zu Fuß, mit dem Fahrrad oder dem öffentlichen Nahverkehr zu erreichen. Die Standorte in zentraler Lage innerhalb der Quartiere wurden von den Organisatoren gewählt, da diese für die Quartiersbewohnerinnen/-bewohner in der Vergangenheit wichtige Orte der Zusammenkunft und des Austausches waren, im Fall des Werksgebäudes sogar mit Produktionsgeschichte. Durch die Symbolik der Orte sollte die Quartiersbevölkerung angesprochen werden, damit nicht nur im Quartier ansässige Betriebe, sondern auch die Nachbarschaft produktiv tätig werden konnte. Das äußere Erscheinungsbild beider Gebäude erweckt allerdings kaum Offenheit. Das Kirchengebäude ist nach innen gewandt und es gibt keine Schaufenster und Sichtbeziehungen nach außen. Lediglich die offene Tür und Licht im Innenraum laden Vorbeigehende zum Eintreten ein. Die Werkhalle ist durch ein kleines Ladenlokal mit einem Schaufenster erreichbar und somit auch nicht direkt einsehbar. Für beide Festivals war zusätzliche Öffentlichkeitsarbeit notwendig, um viele Menschen anzusprechen. Die Aufbereitung der Räumlichkeiten für die Zwischennutzungen ging mit hohem Arbeitsaufwand einher, da beide Gebäude bisher leerstanden und nicht für temporäre Nutzung verfügbar waren. Trotzdem waren die Betreiber mit der Standortwahl zufrieden, im LutherLAB hat sich im Anschluss an das Festival ein Verein gegründet, der die Kirche durch weitere, zum Teil produktive Nutzungen bespielt und weitere kurzfristige Festivals plant.

\section{Inspirierende, offene Produktionsstätten}

Die Store-Factory als Einrichtung der Großunternehmen ist ausdrücklich darauf ausgerichtet, Laufkundschaft anzusprechen und einen spontanen Kundenbesuch zu ermöglichen. Dies wird neben dem Standort in einem Einkaufszentrum auch durch die einem Bekleidungsgeschäft ähnelnde Raum- und Schaufenstergestaltung unterstrichen. Zum Beispiel können Musterpullover mit unterschiedlichen Farben und Schnitten im Vorfeld besichtigt werden. Auch die Fitstation und die Smallest Shoe Factory befinden sich in Sportgeschäften, in denen Prototypen der Einlegesohlen und der Sandalen ausgestellt sind. In der Store-Factory ist zusätzlich eine große Foto-Collage gestaltet, auf der prominente Berliner Influencer und Kunstschaffende bei der Produktion des Pullovers zu sehen sind. Die gesamten Räumlichkeiten der Store-Factory sind auf eine hippe, sportbegeisterte Zielgruppe ausgerichtet: Boden und Wände sind schwarz, im Hintergrund läuft elektronische Musik, in einer Lounge gibt es Bio-Limonade. Die Smallest Shoe Factory ist durch ihre geringe Größe sehr mobil, sodass sie nicht nur im Sporthandel, sondern auch bereits als Blickfang in Schaufenstern und auf einem Design-/Kunstfestival sowie in einer Hochschule für Design installiert wurde (Carballo 2018). Die Fitstation befindet sich in einer vergleichsweise funktionalen Umgebung, in der Testen und Maßnehmen im Vordergrund stehen, damit ein angepasstes Produkt, je nach individueller Gangart, entsteht.

Die Räumlichkeiten aller Fallstudien sind so gestaltet, dass die Produktionsmaschinen und Produktionsprozesse von der Kundschaft beobachtet werden können und transparent sind. Die Produktionsmaschinen sind gut sichtbar in die Verkaufsumgebung integriert bzw. im Fall der Smallest Shoe Factory der Mittelpunkt des Einkaufserlebnisses. In der Store-Factory sind die Strickmaschinen hinter einer Glaswand aufgebaut. Dieser Bereich darf aus Sicherheitsgründen allerdings nur von Fachpersonal betreten werden. Durch die prominente Platzierung der Produktionsanlagen wird Endkunden vermittelt, dass sie Teil des Produktionsprozesses sind, dieser leise, sauber und fair ist, kurze Wege ermöglicht und Lagerhaltung erübrigt. Diese Aufwertung der Produktionsprozesse wird durch die zielgerichtete Nähe zur Kunst- und Designszene im Falle der Store-Factory 
und der Smallest Shoe Factory unterstrichen, durch die Produktion zusätzlich mit einem ästhetischen Narrativ in Verbindung gebracht werden soll.

Die Aufteilung der Geschäftsräume der untersuchten Manufakturen in Produktion und Ausstellung verbindet den Herstellungsprozess und das Endprodukt für Kundinnen und Kunden unmittelbar und transparent. Rohmaterialien wie Früchte, Stoffe, Drähte oder Steine können gesehen und haptisch wahrgenommen werden. Die für die Produktion genutzten Maschinen sind im Vergleich zu denen der oben beschriebenen Großunternehmen bekannte Gebrauchsgegenstände wie Nähmaschinen, Küchengeräte oder Bohrmaschinen, da die handwerklichen Tätigkeiten im Vordergrund stehen.

Produktionsprozesse in den Manufakturen bestehen weniger aus neuen, sauberen Technologien und teilnehmender Beobachtung seitens der Kundschaft. Sie sind durch traditionelle handwerkliche Tätigkeiten, aktive Einbindung der Kundinnen und Kunden in Form von Workshops (siehe Kundenintegration) und die deutlich sichtbare Verarbeitung von Rohmaterialien gekennzeichnet, was je nach Handwerk auch Überschuss, Abfall und Staubentwicklung mit sich bringt. Die Ausstellungsflächen hingegen sind ästhetisch und modern, um die hohe Produktqualität zu unterstreichen. In den Räumlichkeiten der Manufakturen vermischen sich so die Anforderungen einer traditionellen Werkstatt mit denen des gehobenen Einzelhandels. Diese Kombination ist charakteristisch für die untersuchten Produktionsstätten und ein deutlicher Unterschied zu den Großunternehmen, in denen die neuartigen Produktionsprozesse vordergründig sind. Die Gemeinsamkeit beruht auf dem offen einsehbaren und erlebbaren Produktionsprozess, der unmittelbar mit einem Endprodukt verknüpft ist.

Die angemieteten Räumlichkeiten des Makerspace "Dezentrale" umfassen rund $60 \mathrm{qm}$. Das Labor e.V. erstreckt sich auf einer Fläche von rund $100 \mathrm{qm}$. Die Halle 1 nutzt etwa 300 qm. Im Erdgeschoss befinden sich die Produktionsarbeitsplätze und Maschinen, während auf einer Empore Arbeitsplätze und die 3D-Drucker untergebracht sind. Die vorhandene technische Grundausstattung umfasst in allen drei Firmen 3D-Drucker, Scanner, Lasercutter, Lötstation und Computer-Terminals mit entsprechender Open-Source- sowie kommerzieller Software. Mitglieder können sich in allen Räumen frei bewegen, nachdem sie einen Haftungsausschluss unterschrieben haben. Viele Maschinen dürfen nach einer Einführung kostenlos verwendet werden. Die Maschinen, bei denen Kosten durch Abnutzung entstehen, sind gegen Gebühren nutzbar. Zum Befragungszeitpunkt variierten die Gebühren noch stark und wurden häufig als Spenden verbucht. Für Laien besteht die Möglichkeit, zu bestimmten Zeitpunkten an Einführungsworkshops teilzunehmen oder einen Termin mit dem jeweiligen Fachpersonal zu vereinbaren. Zwar bezeichnen sich die untersuchten Werkstätten als offen, da die Maschinen von jeder interessierten Person nach einer Einführung genutzt werden können. Im Vergleich zur Offenheit der Produktionsstätten von Großunternehmen und Manufakturen zielen die Makerspaces aber nicht darauf ab, eine allgemein interessierte Laufkundschaft bzw. Endkunden spontan anzusprechen. Nutzende haben in der Regel bereits im Vorfeld des Besuchs ein bestimmtes Projekt bzw. eine Problemlösung konzipiert, die dann vor Ort umgesetzt und materialisiert wird. Technikaffinität ist hierfür eine notwendige Voraussetzung. Auch die Inspirationsquellen unterscheiden sich. Sie basieren weniger auf Prototypen und bereits vorhandenen Produkten, sondern auf dem Austausch und den Projektbzw. Lösungsvorschlägen, zum Teil auch Exponaten der anderen Nutzenden des Makerspaces. Der Austausch wird durch Begegnungsmöglichkeiten, wie z. B. Gemeinschaftsküchen, unterstützt.

Begegnungsräume wurden auch auf den Festivals angeboten. Interessierte, also Bewohnerinnen und Bewohner des Quartiers oder der Stadt, die den Hauptteil der Nutzerinnen und Nutzer stellten, konnten die Festival-Räumlichkeiten spontan betreten und an Workshops zum Unkostenbeitrag teilnehmen oder zusehen. Weiterhin bestand die Möglichkeit, sich zu Workshops mit begrenzter Teilnehmerzahl anzumelden. Außerdem nutzten Unternehmen die Räumlichkeiten während der Öffnungszeiten als Verkaufs- und Ausstellungfläche. Auch während der Workshops mit Teilnahmebegrenzungen standen die Türen für andere Besucherinnen und Besucher offen, die sich von der geschäftigen Atmosphäre, den Maschinen und den Workshops inspirieren ließen, Wissen erwarben und selbst neue Ideen entwickelten. Den Organisatoren ging es mit dem Do-ityourself-Charakter der Festivals zum einen darum, zum nachhaltigen Umgang mit Materialen und Rohstoffen, z. B. durch Upcycling, anzuregen. Zum anderen verfolgten die Festivals den Zweck, einen Ort des temporären Austauschs im Quartier zu schaffen, an dem die Bewohnerschaft durch gemeinsames Werken und Entwickeln soziale Netzwerke stärken kann. Aus diesem Grund dienten die bereitgestellten Ausstellungsflächen, ähnlich wie in den Produktionsstätten von Großunternehmen und Manufakturen, zwar der Inspiration, allerdings trat der Verkaufszweck im Vergleich dazu deutlich in den Hintergrund. 
Kundenintegration und neue Geschäftsmodelle

In der Store-Factory (Fallstudiengruppe Großunternehmen) erfolgt Kundeninteraktion nach dem Prinzip einer etwa einstündigen Kundenreise, an die die maschinelle Produktion des Pullovers anschließt. Die Reise beginnt mit der Vergabe eines QR-Codes, mit dem spätere Produktionsstufen gespeichert werden können. In einem „Creator-Space“ können Kundinnen und Kunden technikgestützt eigene Muster kreieren und mittels 3D-Bodyscan werden die Körpermaße genommen. An einer „Work Bench“ wählen Kundinnen und Kunden an Computern die endgültige Farbe, Länge und das Muster des Pullovers und versenden den Produktionsauftrag. In diesen Produktionsschritten ist der Wissensaustausch zwischen Kundinnen/Kunden und Produzentinnen/ Produzenten hoch. Kundinnen und Kunden geben ihre Vorlieben preis, während das Produktionsunternehmen durch die gesammelten Daten in Echtzeit lernen kann, welche Farben, Formen und Materialien von Endkunden präferiert werden. Gleichzeitig werden die Kundinnen und Kunden auf der Reise kontinuierlich von Verkaufspersonal begleitet und mittels Wissenstransfer in die einzelnen Arbeitsschritte eingewiesen. Die Fitstation von Hewlett Packard erfasst individuelle Kundenmaße durch eine Ganganalyse elektronisch und übermittelt sie an einen 3D-Drucker, der die Sohlen druckt. In der Smallest Shoe Factory sind Kundinnen und Kunden zwar nicht direkt in den Produktionsprozess eingebunden, sie können aber im Vorfeld den Schuh konfigurieren und bei der nur sechs Minuten andauernden Produktion zusehen. Kundenintegration in den Fallbeispielen erfolgt mit dem Ziel, die individuellen Maße der Kundinnen und Kunden zu nehmen und diese ihre geschmacklichen Vorlieben zum Ausdruck bringen zu lassen (Mass Customization). Die Produktion vor Ort erlaubt eine sehr kurze Markteintrittszeit im Vergleich zu online konfigurierbaren Massenprodukten, wie z. B. Turnschuhen. Kundinnen und Kunden können ihr Produkt sofort im Anschluss mitnehmen und erhalten dadurch eine direkte ,Belohnung für ihren Zeitaufwand während der Vorbereitung des Produktionsprozesses. Darüber hinaus gelingt es, die Produkte durch die intensive Einbindung der Kundinnen und Kunden zu emotionalisieren, da sie nun mit einem (positiven) Erlebnis und persönlichen Entscheidungen in Verbindung stehen. Dass beteiligte Kundinnen und Kunden gleichzeitig ihre Daten preisgeben und unentgeltliche Arbeit leisten, rückt dabei in den Hintergrund.

In den drei Manufakturen (Marmeladenmanufaktur, Textilwerkstatt Liebesgruß und Schmuckschmiede) sind Kundenworkshops eine zentrale Säule der jewei- ligen Geschäftsmodelle und ein wichtiges Instrument der Kundenintegration und Kundenbindung. Dazu sind ausreichend Arbeitsplätze in den Geschäftsräumen vorhanden. In mehrstündigen Workshops werden Produkte unter Anleitung von Teilnehmenden gegen Gebühr selber hergestellt. Daneben besteht auch die Möglichkeit des Direktverkaufs vor Ort. Spontane Koproduktion ist nicht möglich, da die Workshops nur nach vorheriger Anmeldung, zu bestimmten Zeiten und bei einer ausreichenden Zahl an Interessentinnen und Interessenten stattfinden. Mit derartigen Workshops erfährt die im Einzelhandel übliche Selbstbedienung eine Weiterentwicklung hin zu Eigenproduktion, da Kundinnen und Kunden ihre Produkte im Rahmen vorgegebener Produktionsmittel gestalten und herstellen. Während Kundinnen und Kunden in den beschriebenen Produktionsprozessen der Großunternehmen eine aktive Rolle in der Vorbereitung der Produktion haben, die Produktion aber maschinell stattfindet, leisten sie in den Manufakturen den entscheidenden Beitrag im Herstellungsprozess selbst. Die Anleitung zur Eigenproduktion und das Vermitteln von handwerklichen Fähigkeiten in den Workshops ist eine angebotserweiternde Dienstleistung der Manufakturen. Gleichzeitig ist sie aber auch eine Möglichkeit, das Produktspektrum durch Ideen, die von Kundinnen und Kunden auf den Workshops kommuniziert werden, zu verbessern oder zu erweitern. Die Lerneffekte im Rahmen der Produktionsstufe der Fertigung sind daher gegenseitig.

In FabLabs liegt der Schwerpunkt vor allem auf dezentraler Fertigung mittels des genannten Technikequipments. Die "Dezentrale“ bietet unterschiedliche ,Labs' für Interessierte an. So befasst sich das e:Lab - Bürgerlabor für Energieinnovationen mit neuartigen Konzepten zur Energieerzeugung, -speicherung, -verteilung und -nutzung. Und im BioLab geht es um das Potenzial biologischer Prozesse in Lebensmittelproduktion, Materialentwicklung und Abfallnutzung. Während der Öffnungszeiten bieten Mitarbeiterinnen und Mitarbeiter der "Dezentrale“ Unterstützung bei Projektrealisierungen. Zusätzlich finden regelmäßig Workshops zu unterschiedlichen Themen statt. Ziel der "Dezentrale“ ist es, ein Umfeld zu bieten, in dem sich Ideen zu wirtschaftlich verwertbaren Produkten entwickeln können. Auch die Halle 1 möchte Ausgründungen im wirtschaftlichen Bereich fördern und wurde gezielt zur Prototypen-Entwicklung an der Hochschule angesiedelt. Die Do-it-yourself-Kultur des Personals vor Ort ist dabei ausschlaggebend. Zusätzlich werden gemeinsam mit weiteren Einrichtungen und Initiativen Kurse für Schüler/innen 
in MINT-Fächern ${ }^{6}$ angeboten. Diese sollen begeistert werden, Produkte selbst zu kreieren und zu reparieren und Erfindergeist zu entwickeln. Die Öffnungszeiten des Labors werden auf der Homepage durch einen Labor Status Bot bekannt gegeben, der sich je nach Status des Hauptstromschalters am Eingang des Labors ändert. In erster Linie steht das Labor für die Vereinsmitglieder bereit. Andere Personen können die Räumlichkeiten projektbedingt, zu Workshops, zum Repair Café oder zu Aktionen (meist) kostenlos nutzen. In Makerspaces ist die Unterscheidung zwischen Kundschaft und der durch Verkaufspersonal oder Geschäftsinhaberinnen/ -inhaber repräsentierten Anbieterseite nicht angemessen. Die dort aktiven Personen sind Prosumenten, die Produkte für den eigenen Konsum herstellen. Es gibt zwar organisatorische Strukturen (Vereinsvorstand, Geschäftsführung oder ähnliches), diese zeichnen aber für die Wartung der Maschinen, für Kostenausgleich und Raummiete verantwortlich und nicht für die von der Nutzerschaft hergestellten Produkte. Das in Makerspaces ausgetauschte Wissen kann sich auf alle Produktionsstufen beziehen. Unterschiede zwischen Nutzer- und Produzentenwissen gibt es nicht, Variationen können aber durch die unterschiedlichen Erfahrungen der vor Ort wechselnden Nutzerschaft entstehen.

Besucherinnen und Besucher der Festivals wurden direkt in den Produktionsprozess integriert, indem sie sich selbst vorgefertigte Rohmaterialen, z. B. aus Holz oder Stoff, aussuchen und unter Anleitung in Koproduktion zusammenbauen konnten. Dabei war es möglich, zwischen unterschiedlichen Produkten und Anbietern spontan frei zu wählen. Der Wissensaustausch fand im Vergleich zu Makerspaces begrenzt statt und bezog sich auf die Produktionsstufe des Zusammenfügens. Er diente der Befähigung der Teilnehmenden, wobei auch hier unternehmensseitig die Möglichkeit bestand, aus Kundenwissen und -präferenzen zu lernen. Durch die vorgegebene Auswahl an Materialien und Produkten war die Kundenintegration bei der Entwicklung gering, erhöhte sich im Produktionsprozess dann im Rahmen der Fertigung und des Zusammenfügens. Im Gegenzug generierten die Workshop-Anbieter Wissen über aktuelle Trends durch die Kundinnen und Kunden und entwickelten in Teilen die Produkte mit spezifischem Kundenwissen weiter. Der Austausch zwischen Personen, die Workshops geleitet haben, führte in Einzelfällen zu weiteren Kooperationen. Für die Unternehmen waren die Festivals eine zusätzliche, außergewöhnliche Verkaufsumgebung mit hoher Besucherzahl, die der Unter-

6 Mathematik, Informatik, Naturwissenschaft, Technik nehmenspräsentation und Kundenakquise diente. Die Orte und die begrenzte Dauer der Festivals vermittelten eine besondere Atmosphäre, durch die die ausgestellten Produkte und angebotenen Dienstleistungen seitens der Festivalbesucher mit einem Erlebnis verbunden wurden. Ähnlich der Fälle in den Großunternehmen und Manufakturen war ein Teilaspekt der Festivals die Emotionalisierung der Produkte. Gleichzeitig sollte den Kundinnen und Kunden vermittelt werden, welche Unternehmen in ihrem Quartier ansässig sind und welche Produkte lokal hergestellt werden. Die Festivalbesucherinnen und -besucher wurden daher sowohl als Kundinnen und Kunden als auch als Bewohnerschaft des Quartiers angesprochen.

\section{Ergebnisse}

Die vier Fallstudiengruppen Großunternehmen, Manufakturen, offene Werkstätten und Festivals sind Beispiele für produktionsbedingte temporäre räumliche Nähe und für das Verschmelzen von Produktion und Dienstleistung im Rahmen urbaner Produktion. Die Interaktion zwischen Produzentinnen/Produzenten und Kundinnen/Kunden dauert wenige Minuten bis Stunden und ist durch intensive Austauschprozesse (Erlebniskauf) geprägt. Diese haben das Ziel, ein individualisiertes Endprodukt, zum Teil in Eigenproduktion, zu fertigen. Dafür ist der Austausch von Wissen notwendig, durch den unternehmerseitig das Herstellungsverfahren vermittelt und kundenseitig die geschmacklichen Vorlieben und Maße preisgegeben werden.

Der Grad der Kundenintegration und damit die Intensität produktionsbedingter temporärer räumlicher Nähe im Produktionsprozess der vier Fallgruppen ist unterschiedlich, wie in Abbildung 1 dargestellt wird. In Großunternehmen ist die Kundenintegration in den Produktionsphasen Entwicklung und Materialauswahl tendenziell höher als in den maschinell erfolgenden Phasen der Fertigung und des Zusammenfügens. Dies ist bei Festivals umgekehrt, da dort oft mit vorgefertigten Bauteilen gearbeitet wird. In Makerspaces hingegen ist Kundenintegration aufgrund des Prosumptions-Ansatzes von Beginn an sehr hoch, kann in späteren, maschinellen Produktionsphasen (z. B. durch 3D-Druck) aber abnehmen. Die Kundenintegration bei Manufakturen ist im gesamten Prozess gleichbleibend hoch.

Zusätzlich geht es aber auch um den konkreten handwerklichen Produktionsprozess. Dadurch verändern sich die Orte der Produktion, welche in diesen Fällen nun dort stattfindet, wo viele Endkunden sind: 


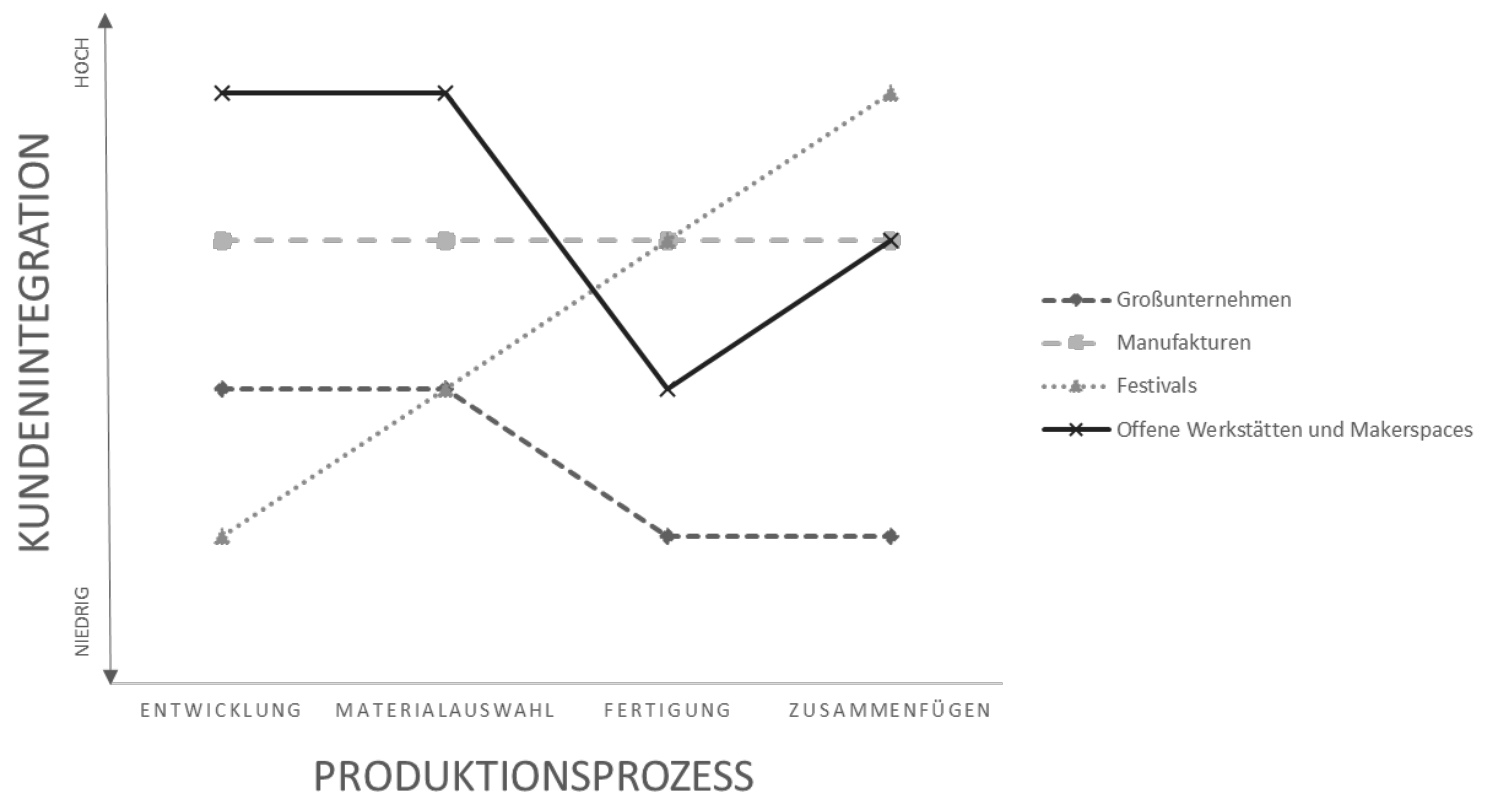

Abbildung 1: Kundenintegration im Produktionsprozess

in Einzelhandelslagen, Wohngebieten und Stadtteilzentren. Neue Technologien und Minifabriken tragen dazu bei, dass Produktion auf geringer Fläche möglich wird. Während für Großunternehmen frequentierte Einzelhandelsstandorte eine große Rolle spielen, um eine hohe Aufmerksamkeit zu erzielen, befinden sich die Manufakturen in urbanen Szenevierteln. Standorte von Makerspaces und Festivals werden nach Bedarf (Betreiberin/Betreiber) oder Interesse (Zielgruppe) gewählt. Die Ansiedlung in einem lebendigen, urbanen Umfeld als neuem Produktionsstandort ist bedeutend für die Betriebe, um Nähe zu Kundinnen und Kunden aufzubauen. Ein Standort außerhalb der Innenstadt in einem klassischen Gewerbegebiet käme daher nicht in Frage. In den Fallbeispielen der "Dezentrale“ und einiger Manufakturen wurden von den Geschäftsinhaberinnen und -inhabern außerdem die Nähe zum Wohnstandort als wichtiges Kriterium für die Wahl des Unternehmensstandortes und die Vernetzungsmöglichkeiten zu anderen, im Quartier ansässigen Unternehmen und Initiativen hervorgehoben. Bei den untersuchten FabLabs kommt zwar ein Großteil der Nutzerinnen und Nutzer aus einem universitären Umfeld, als offene Werkstatt sollen aber auch andere Nutzergruppen angesprochen werden. Deshalb wurde in zwei der drei Fälle eine innerstädtische Lage bevorzugt.

Tabelle 1 fasst die Ergebnisse des Beitrags anhand der Fallgruppen Großunternehmen, Manufakturen, Makerspaces und Festivals zusammen. Es ist deutlich, dass produktionsbedingte temporäre räumliche Nähe mit neuen Geschäftsmodellen einhergeht, die Kundeninteraktion ermöglichen. Die Großunternehmen setzen auf Mass Customization, indem durch Maschinen vor Ort direkt ein individualisiertes Produkt hergestellt wird. Durch die von Adidas offerierte Kundenreise lernt das Unternehmen zudem von den Daten, die Kundinnen und Kunden an den jeweiligen Stationen hinterlassen: Größe, Vorlieben für Modelle und Farben. Die DesignLeistung wird an die Kundschaft abgegeben. Zudem profitieren die Großunternehmen vom Produktionserlebnis, das der Marke einen innovativen Charakter durch eine neue Form des Einkaufens verleiht. Bei Manufakturen ist der spontane Einbezug der Kundschaft meist nicht möglich, da sie ihre Workshops auf Anfrage anbieten. Dort werden die eigenen Produkte im Eingangsbereich des Ladenlokals präsentiert. Durch die Eigenproduktion soll die Wertschätzung gegenüber den Produkten erhöht und eine positive Verbindung zu den Produkten aufgebaut werden, um so den Absatz zu steigern. Während bei den Großunternehmen und Manufakturen die Prozesse, Materialien und Muster für die Produktion vorgegeben werden, erwarten Makerspaces und Festivals höheres Engagement und Eigenverantwortung der Kundschaft, eigene Projekte mitzubringen und vor Ort umzusetzen.

Nicht nur die Lage in der Stadt ändert sich mit neuen Produktionsformen, sondern auch die Produktionsstätten. Häufig sind die Räumlichkeiten in einen allgemeinen Geschäftsbereich mit Kundenempfang, in dem die (Vor-) 
Tabelle 1: Produktionsbedingte räumliche Nähe anhand der Fallgruppen

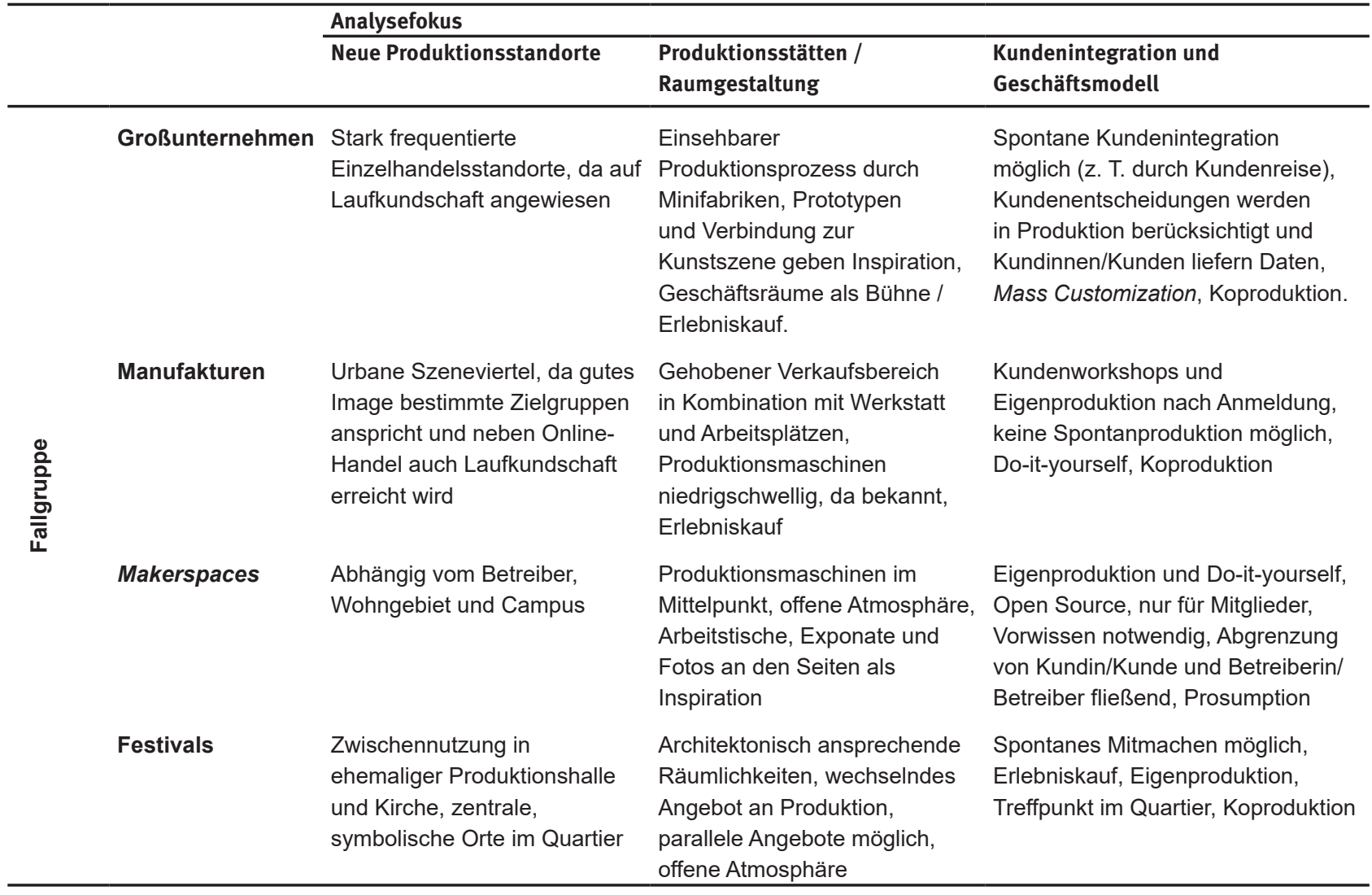

Produkte haptisch wahrgenommen werden können, und einen Werkstatt-/Produktionsbereich, der jedoch nicht immer öffentlich zugänglich ist, aufgeteilt. Die Offenheit, das heißt die Frage, wann die Produktionsstätten (unter Anleitung) genutzt werden können und von wem, ist kaum begrenzt. Während Makerspaces und offene Werkstätten sowie Manufakturen zu allgemeinen Öffnungszeiten betreten werden können, fanden das Festival der Urbanen Produktion und die Test-/Pilotierungsmaßnamen Factory-Store, Fitstation und Smallest Shoe Factory nur temporär statt. Es überlagern sich dadurch unterschiedliche Temporalitäten. Während ein Festival oder die Store-Factory an einem spezifischen Standort auf befristete Dauer eingerichtet ist, nimmt der gemeinsame Produktionsprozess mit den Teilnehmenden oder Kundinnen/Kunden hingegen nur wenige Stunden in Anspruch. Die untersuchten Manufakturen und Makerspaces sind dauerhaft angelegt und es besteht die Möglichkeit, zu Workshop-/Öffnungszeiten auch über einen längeren Zeitraum (z. B. über mehrere Tage oder Wochen) in den Produktionsprozess eingebunden zu werden. Kundinnen und Kunden sind dann von Beginn an in den Produktionsprozess involviert, entscheiden über die zu verwendenden Materialen und nehmen direkt Einfluss auf das Produkt und dessen Gestaltung.

\section{Fazit}

In diesem Beitrag wird argumentiert, dass temporäre räumliche Nähe einen produktionsbedingten Zweck verfolgen kann. Temporäre räumliche Nähe ist damit nicht nur ein wichtiges Instrument des Wissensaustauschs innerhalb von Geschäftsbeziehungen, sondern dient ebenso der temporären Einbeziehung von Endkunden zwecks individualisierter Produktion von Konsumgütern. Im Rahmen der Interaktion von Produzentin/Produzent und Kundin/Kunde finden neben Wissensaustausch auch Produktionstätigkeiten statt, wodurch produktionsbedingte temporäre räumliche Nähe eine marktnahe Ergänzung zu den etablierten US-amerikanischen und asiatischen Produktionsstandorten ist. Für die etablierte Forschung zu temporärer räumlicher Nähe bedeutet dies, zukünftig auch Endkunden, urbane Standorte und neue Produktionsprozesse als Forschungsgegenstände zu berücksichtigen. 
Produktionsbedingte temporäre räumliche Nähe hat einen deutlichen Dienstleistungscharakter. Kundinnen und Kunden werden bei der Herstellung ihrer Güter von Fachpersonal angeleitet, inr Aufenthalt wird als Reise interpretiert, es bestehen intensive Interaktionen zwischen Produzent und Endkunde. Interessierte Kundinnen und Kunden können die Geschäfte betreten und produktiv tätig werden, sich zu Workshops anmelden oder sie sind Mitglied des betreibenden Vereins, z. B. eines Makerspaces, und haben Zugang zum benötigten technischen Equipment. Dadurch können die produzierenden Firmen in Echtzeit auf Kundenwünsche eingehen, die Lagerhaltung der Güter entfällt. Wie beim Konsum von Dienstleistungen ist die Kundin/der Kunde in den Geschäftsräumen bzw. in Kontakt mit den Anbietern der Dienstleistung, bevor das Produkt gemeinsam hergestellt wird. Insofern ist es folgerichtig, dass neue Produktionsstandorte dort entstehen, wo viele Endkunden anzutreffen sind. Der kurze Produktionsprozess findet im Vergleich zu massenhaft hergestellten Konsumgütern spontan statt. Dies hat - wenn auch in geringem Umfang - Auswirkungen auf Wertschöpfungsketten, da Wertschöpfungsstufen räumlich verlagert werden. Produktion findet in den Fällen der Großunternehmen nicht mehr unabhängig und räumlich getrennt vom Verkauf statt, sondern es wird eine vorgelagerte Wertschöpfungsstufe in den Verkauf integriert und zum Teil umgekehrt. Das heißt, Kundinnen und Kunden treffen eine Kaufentscheidung, bevor die in der Produktion generierte Wertschöpfung vollzogen ist, um im Anschluss an ihre Kaufentscheidung aktiver Teil der Wertschöpfung zu werden.

Produktionsbedingte temporäre räumliche Nähe zwischen Produzentinnen/Produzenten und Kundinnen/Kunden und damit verbundene urbane Produktion werden die massenhafte Herstellung von Konsumgütern nicht ablösen. Sie sind jedoch ein wichtiger Faktor, der die Wahl von Geschäftsmodellen und Unternehmensstandorten angesichts des gegenwärtigen Trends der Individualisierung von Konsumgütern beeinflussen wird. Produktionsbedingte temporäre räumliche Nähe könnte somit Einzelhandelsstandorte durch die induzierte Kundenbindung wieder attraktiv machen.

\section{Literatur}

Balland, P.-A.; Boschma, R.; Frenken, K. (2015): Proximity and Innovation: From Statics to Dynamics. In: Regional Studies 49, 6, 907-920. doi: 10.1080/00343404.2014.883598
Bathelt, H. (2017): Trade Fairs and Innovation. In: Bathelt, H.; Cohendet, P.; Henn, S.; Simon, L. (Hrsg.): The Elgar Companion to Innovation and Knowledge Creation. Cheltenham, 509-522.

Bathelt, H.; Henn, S. (2014): The geographies of knowledge transfers over distance: toward a typology. In: Environment and Planning A 46, 6, 1403-1424. doi: 10.1068/a46115

Bieger, T.; Reinhold, S. (2011): Das wertbasierte Geschäftsmodell - Ein aktualisierter Strukturierungsansatz. In: Bieger, T.; zu Knyphausen-Aufseß, D.; Krys, C. (Hrsg.): Innovative Geschäftsmodelle. Konzeptionelle Grundlagen, Gestaltungsfelder und unternehmerische Praxis. Wiesbaden, 13-70. doi: 10.1007/978-3-642-18068-2

Boschma, R. (2005): Proximity and Innovation: A Critical Assessment. In: Regional Studies 39, 1, 61-74. doi: 10.1080/0034340052000320887

Brandt, M.; Butzin, A.; Gärtner, S.; Meyer, K.; Hennings, G.; Siebert, S.; Ziegler-Hennings, C. (2017): Produktion zurück ins Quartier? Neue Arbeitsorte in einer gemischten Stadt. Endbericht im Auftrag des Ministeriums für Heimat, Kommunales, Bau und Gleichstellung des Landes NordrheinWestfalen. Gelsenkirchen.

Brinks, V.; Ibert, O. (2015): Mushrooming Entrepreneurship: The Dynamic Geography of Enthusiast-Driven Innovation. In: Geoforum 65, 363-373. doi: 10.1016/j.geoforum.2015.01.007

Carballo, C. (2018): Here's How the 'World's Smallest Shoe Factory' Produces Custom Shoes in Six Minutes. In: Footware News. https://footwearnews.com/2018/focus/athletic-outdoor/keenuneekbot-shoes-future-robotic-manufacturing-1202562504/ (10.09.2019).

Cohen, J.; Jones, W.M.; Smith, S.; Calandra, B. (2017): Makification: Towards a Framework for Leveraging the Maker Movement in Formal Education. In: Journal of Educational Multimedia and Hypermedia 26, 3, 217-229.

Drengner J.; Jahn S. (2012): Konsumerlebnisse im Dienstleistungssektor - Die Konzeptualisierung des Erlebniskonstrukts am Beispiel kollektiv-hedonistischer Dienstleistungen. In: Bruhn, M.; Hadwich, K. (Hrsg.): Customer Experience. Wiesbaden, 227-250. doi: 10.1007/978-3-83494001-8

Drotschmann, M. (2010): Baumarkt 2.0. Do-It-Yourself, Youtube und die Digital Natives. In: Journal of New Frontiers in Spatial Concepts 2, 18-27.

Ematinger, R. (2018): Von der Industrie 4.0 zum Geschäftsmodell 4.0. Chancen der digitalen Transformation. Wiesbaden. doi: 10.1007/978-3-658-19474-1

Erbstößer, A.-C. (2016): Produktion in der Stadt. Berliner Mischung 2.0. Berlin.

Flögel, F.; Zademach, H.-M. (2017): Bank branches as places of knowledge creation: Conceptual considerations and empirical findings at the micro-geographical scale. In: Erdkunde 71, 4, 301-312. doi: 10.3112/erdkunde.2017.04.03

Følstad, A.; Kvale, K. (2018): Customer Journeys: a Systematic Literature Review. In: Journal of Service Theory and Practice 28, 2, 196-227. doi: 10.1108/JSTP-11-2014-0261

Fox, S. (2013): Paradigm shift: Do-it-yourself (DIY) Invention and Production of Physical Goods for Use or Sale. In: Journal of Manufacturing Technology Management 24, 2, 218-234. doi: 10.1108/17410381311292313

Grabher, G.; Ibert, O.; Flohr, S. (2008): The Neglected King: The Customer in the New Knowledge Ecology of Innovation. In: 
Economic Geography 84, 3, 253-280. doi: 10.1111/j.19448287.2008.tb00365.x

Growe, A. (2018a): Buzz at workplaces in knowledge-intensive service production: Spatial settings of temporary spatial proximity. In: European Urban and Regional Studies. doi: 10.1177/0969776418784999

Growe, A. (2018b): Developing Trust in Face-to-Face Interaction of Knowledge Intensive Business Services (KIBS). In: Regional Studies. doi: 10.1080/00343404.2018.1473567

Henn, S.; Bathelt, H. (2015): Knowledge Generation and Field Reproduction in Temporary Clusters and the Role of Business Conferences. In: Geoforum 58,104-113. doi: 10.1016/j. geoforum.2014.10.015

Hirshberg, P.; Dougherty, D.; Kadanoff, M. (2016): Maker City: A Practical Guide for Reinventing American Cities. San Francisco.

Huba, J.; McConnell, B. (2006): Citizen Marketers: When People Are the Message. Chicago.

Humphreys, A.; Grayson, K. (2008): The Intersecting Roles of Consumer and Producer: A Critical Perspective on Co-production, Co-creation and Prosumption. In: Sociology Compass 2, 3, 963-980. doi: 10.1111/j.1751-9020.2008.00112.x

Kunz, W. H.; Mangold, M. (2004): Hybride Communities als Treiber des Kundenwertes. In: Herstatt, C.; Sander, J. G. (Hrsg.): Produktentwicklung mit virtuellen Communities. Kundenwünsche erfahren und Innovationen realisieren. Wiesbaden, 69-98. doi: 10.1007/978-3-322-84540-5

Läpple, D. (2013): Produktion zurück in die Stadt? In: Kronauer, M.; Siebel, W. (Hrsg.): Polarisierte Städte. Soziale Ungleichheit als Herausforderung für die Stadtpolitik. Frankfurt am Main, 129-150.

Lampel, J.; Meyer, A. D. (2008): Field-Configuring Events as Structuring Mechanisms: How Conferences, Ceremonies, and Trade Shows Constitute New Technologies, Industries, and Markets. In: Journal of Management Studies 45, 6, 1025-1035.

Lemon, K. N.; Verhoef, P. C. (2016): Understanding Customer Experience Throughout the Customer Journey. In: Journal of Marketing 80, 6, 69-96. doi: 10.1509/jm.15.0420

Lundvall, B.-A. (1992): User-Producer Relationships, National Systems of Innovation and Internationalisation. In: Lundvall, B.-A. (Hrsg.): National Systems of Innovation: Towards a Theory of Innovation and Interactive Learning. London, 47-70.

Meier, N.; Wirth, M. (2013): FabLabs - High-Tech-Werkstätten für Jedermann. Würzburg. = CEDIFA-Arbeitsbericht 4.

Mikunda, C. (2006): Brand Lands, Hot Spots and Cool Spaces: Welcome to the Third Place and the Total Marketing Experience. London.

Moritsch, H. S. (2016): Design, Handwerk und urbane Produktion. In: Wirtschaftsagentur Wien (Hrsg.): Focus Crafted in Vienna Wien produziert. Kreative Geschäftsmodelle, Strategien und Konzepte für städtische Produktion. Wien, 29-31.

Mühl, C.; Busch, H.-C.; Fromhold-Eisebith, M.; Fuchs, M. (2019): Urbane Produktion. Dynamisierung stadtregionaler Arbeitsmärkte durch Digitalisierung und Industrie 4.0? Düsseldorf. $=$ FGW-Studie Digitalisierung von Arbeit 14 .

Nadeau, R. (2006): Living Brands: Collaboration + Innovation = Customer Fascination. New York.

o.V. (2018a): FitStation will Running revolutionieren. In: SAZSport. https://www.sazsport.de/hersteller/brooks/fitstation-runningrevolutionieren-1471313.html (10.09.2019).
o.V. (2018b): „MakerSpace“ erhält mehr als 1,6 Millionen Euro Fördermittel. In: Trikon. Nachrichten aus der Westfälischen Hochschule 5, 12.

Pavitt, K. (1984): Sectoral Patterns of Technical Change: Towards a Taxonomy and a Theory. In: Research Policy 13, 6, 343-373. doi: 10.1016/0048-7333(84)90018-0

Pine, B. J.; Gilmore, J. H. (1998): Welcome to the Experience Economy. In: Harvard Business Review 76, 4, 97-105.

Pine, B. J.; Gilmore, J. H. (2000): Erlebniskauf: Konsum als Ereignis, Business als Bühne, Arbeit als Theater. München.

Reichwald, R.; Piller, F. (2009): Interaktive Wertschöpfung. Open Innovation, Individualisierung und neue Formen der Arbeitsteilung. Wiesbaden. doi: 10.1007/978-3-8349-9440-0

Scheelhaase, T.; Zinke, G. (2016): Potenzialanalyse einer zirkulären Wertschöpfung im Land Nordrhein-Westfalen. Düsseldorf.

Schmidt, S.; Ibert, O.; Kuebart, A.; Kühn, J. (2016): Open Creative Labs in Deutschland. Typologisierung, Verbreitung und Entwicklungsbedingungen. Erkner.

Schüßler, E.; Grabher, G.; Müller-Seitz, G. (2015): Field-Configuring Events: Arenas for Innovation and Learning? In: Industry and Innovation 22, 3, 165-172. doi: 10.1080/13662716.2015.1038098

Thallmaier, S. R. (2015): Customer Co-Design. A Study in the Mass Customization Industry. Wiesbaden. doi: 10.1007/978-3-65807526-2

Toffler, A. (1981): The Third Wave. London.

Torre, A.; Rallet, A. (2005): Proximity and Localization. In: Regional Studies 39, 1, 47-59. doi: 10.1080/0034340052000320842

Urry, J. (2007): Mobilities. Cambridge.

Verhoef, P. C.; Lemon, K. N.; Parasuraman, A.; Roggeveen, A.; Tsiros, M.; Schlesinger, L. A. (2009): Customer Experience Creation: Determinants, Dynamics and Management Strategies. In: Journal of Retailing 85, 1, 31-41. doi: 10.1016/j. jretai.2008.11.001

Welzbacher, C.; Pirk, W.; Ostheimer, A.; Bartelt, K.; Bille, J.; Klemmt, M. (2015): Digitalisierung der Wertschöpfungs- und Marktprozesse. Herausforderungen und Chancen für das Handwerk. Eine Vorstudie im Rahmen der Konzeption eines Demonstrations- und Kompetenzzentrums im Handwerk. Hannover.

Wolf, M.; McQuitty, S. (2011): Understanding the do-it-yourself consumer: DIY motivations and outcomes. In: Academy of Marketing Science Review 1, 3-4, 154-170. doi: 10.1007/s13162011-0021-2

Xie, C.; Bagozzi, R. P.; Troye, S. V. (2008): Trying to Prosume: Toward a Theory of Consumers as Co-creators of Value. In: Journal of the Academy of Marketing Science 36, 1, 109-122. doi: 10.1007/s11747-007-0060-2

Yin, R. K. (2003): Case Study Research. Design and Methods. Thousand Oaks.

Zentes, J. (2012): Vertikale Integration. In: Zentes, J.; Swoboda, B.; Morschett, D.; Schramm-Klein, H. (Hrsg.): Handbuch Handel. Strategien - Perspektiven - Internationaler Wettbewerb. Wiesbaden, 89-101. doi: 10.1007/978-3-8349-3847-3 